\title{
CHROMOSPHERIC FINE STRUCTURES \\ NEAR THE SOLAR LIMB IN Ha
}

\author{
RAWI BHAVILAI \\ Dept. of Physics, Faculty of Science, Chulalongkorn University, Bangkok 5, Thailand
}

\begin{abstract}
The solar limb has been studied visually and photographically in the light of $\mathrm{H} \alpha$ and the combined results are reported. The appearance of a spicule changes with wavelength settings. Effects due to changes of spicule velocities with height and opacitywavelength relations are indicated. A number of bright spicules seen in the wings are identified with bright mottles seen in the line core. The dark band appears between $\Delta \lambda= \pm 1.0 \AA$ and $\Delta \lambda= \pm 0.70 \AA$. Examination of a small mottle cluster in a series of photographs taken in steps from $\mathrm{H} \alpha+0.85 \AA$ to $\mathrm{H} \alpha+0.10 \AA$ leads to a three-dimensional model of a typical mottle cluster.
\end{abstract}

\section{DISCUSSION}

(i) On the Magnetic Field Configuration in the Dark Mottles

Meyer remarked that the configuration of the magnetic field associated with the structures shown in the figures seems a little bit strange. Can most dark mottles be described as such a curved, prominence type phenomena (Beckers)? Yes, this is the common, not the exceptional behaviour (Bhavilai).

(ii) The Inclination of the Feet of the Dark Mottles

The feet of the dark mottles, or the parts closest to the rosette center, generally appear straight since the region where the mottle curves around coincides with the region where it becomes diffuse and therefore transparent (Bhavilai).

Schmidt asked about the inclination, or steepness, of the feet of the dark mottles. The inclination is more or less the same as that for the bright mottles (Bhavilai). 